\title{
Caracterización de procesos cognitivos de memoria, lenguaje y pensamiento, en estudiantes con bajo y alto rendimiento académico*
}

\section{Characterizing Cognitive Processes: Memory, Language and Thought in Students with Low and High Academic Achievement}

\author{
Edilberto Mejía Quintero \\ Universidad Católica de Colombia \\ Hugo Escobar Melo** \\ Pontificia Universidad Javeriana, Colombia
}

Recibido: 30 de julio de 2011 Revisado: 15 de octubre de 2011 Aceptado: 7 de diciembre de 2011

\section{Resumen}

Este artículo presenta la caracterización de los procesos de memoria, lenguaje y pensamiento de cuatro grupos de estudiantes con bajo, medio, alto y superior rendimiento académico, entre los años de primero y quinto de primaria, con un rango entre seis y once años de edad, de un centro educativo de Bogotá-Colombia. Se aplicaron diez sub-pruebas del WISC-IV, para describir dichos procesos. El rendimiento académico se corroboró mediante informes escolares. Los resultados se procesaron con el SPSS-19, mediante ANOVA, y DHS de Tukey. El aspecto más influyente en las calificaciones es la comprensión verbal con una correlación de .718; le sigue el razonamiento perceptual con una correlación de .659; y por último la memoria de trabajo con una correlación de .577.

Palabras clave: logro académico [SC 00190], aprendizaje [SC 28030], problemas de aprendizaje [SC 27990], desarrollo [SC 13830], memoria [SC 30570], lenguaje [SC 27740], pensamiento [SC 52880].

* $\quad$ Artículo de investigación producto de la actividad conjunta en la linea de investigación, evaluación y desarrollo de Procesos congnitivos.

** Correspondencia: Edilberto Mejía Quintero, Universidad Católica de Colombia. Correo electrónico betomeq@yahoo.es. Hugo Escobar Melo, Pontificia Universidad Javeriana. Dirección postal: Cra 5 No 39 - 00, Piso 1. Bogotá D.C. - Colombia. Correo electrónico: escobarh@javeriana.edu.co 


\section{Abstract}

This paper presents a characterization of the processes of memory, language and thought of four groups of students with low, medium, high and higher academic achievement among first and fifth grade, aged between 6 and 11 years, in an educational center in Bogotá-Colombia. to 10 sub-tests of the WISC-IV Were applied, to describe these processes. Academic performance was corroborated by school reports. The results were processed using SPSS-19, ANOVA and Tukey's DHS. The most influential aspect is the verbal comprehension scores with a correlation of .718; followed by perceptual reasoning with a correlation of .659 , and finally the working memory with a correlation of .577 .

Keywords: academic achievement [SC 00190], learning [SC 28030], learning problems [SC 27990], development [SC 13830], memory [SC 30570], language [SC 27740], thought [SC 52880].

\section{Introducción}

En todo proceso educativo se presentan diferencias entre los estudiantes; mientras algunos se adaptan fácilmente a los programas educativos y responden satisfactoriamente, otros presentan grandes dificultades para asimilar los conocimientos que brindan los planes académicos ofrecidos por la institución educativa, la primera situación queda evidenciada en el alto rendimiento académico, mientras que la segunda se constata con el bajo rendimiento académico de los estudiantes que presentan este tipo de dificultad.

El rendimiento académico es el nivel de conocimiento adquirido en determinadas materias o áreas educativas comparando la edad y el grado o nivel escolar. En él influyen muchos factores como: el estrato socioeconómico, los programas de estudio, las metodologías usadas en la enseñanza, el nivel de pensamiento de los alumnos (Benítez, Giménez y Osicka, 2000), los factores familiares y personales (Ivanovich y Ivanovich, 1988), el grupo de pares, el contexto educativo y las relaciones interpersonales.

Generalmente se mide el rendimiento académico mediante las calificaciones escolares. Cascón (2000) en su estudio "análisis de las calificaciones escolares como criterio de rendimiento académico" afirma:
Prácticamente en todos los países desarrollados y en vías de desarrollo, las calificaciones escolares han sido, son y probablemente seguirán siendo el indicador del nivel educativo adquirido y que a su vez estas son reflejo de las evaluaciones y/o exámenes donde el alumno ha de demostrar sus conocimientos sobre las distintas áreas o materias, que el sistema considera necesarias y suficientes para su desarrollo como miembro activo de la sociedad (Cascón, 2000, pp. 1-11).

Idea que es corroborada por Navarro (2003) quien afirma que algunos criterios tenidos en cuenta para determinar el rendimiento académico han sido la evaluación escolar, las calificaciones del alumno, el nivel de inteligencia, el desempeño individual del estudiante y la manera como es influido por el grupo de pares, el aula o el propio contexto educativo.

Según Zapata, L.; De Los Reyes, C.; Lewis, S. \& Barceló, E. (2009), el rendimiento académico puede ser una variable difícil de medir debido a su carácter multifactorial. Dichos autores retoman a Macizo (2006) y agregan que, esto sucede igualmente con la memoria de trabajo, ya que requiere de diferentes recursos cognitivos como la atención y la flexibilidad cognitiva, entre otros.

En el marco general de la educación, Niebla (2007) afirma que coadyuvar en el logro de un excelente rendimiento académico genera estudiantes con conocimientos que les permiten enfrentar 
los retos individuales y sociales que demanda la sociedad.

En esta perspectiva, el rendimiento académico es el resultado de múltiples factores que se cruzan en un momento y en un sujeto particular (Paba, Lara \& Palmezano, 2008).

En esta investigación para determinar quiénes tienen bajo, medio, alto o superior rendimiento académico, se tuvieron en cuenta los informes respectivos de la institución educativa donde se realizó la investigación en términos de las calificaciones que oscilan entre 10 y 67 (bajo), 68 y 83 (medio), 84 y 94 (alto) y entre 95 y 100 (superior).

En el rendimiento académico influyen, de alguna manera, los procesos cognitivos de memoria, lenguaje y pensamiento; así por ejemplo, Betancourt \& González afirman que: "tradicionalmente se ha señalado la atención, la memoria, el pensamiento y el lenguaje como procesos cuyo insuficiente desarrollo provoca déficit en el aprendizaje" (2003, p. 31).

En esta indagación son importantes los procesos cognitivos de memoria, lenguaje y pensamiento, por tal motivo, a continuación se plantean algunos aspectos fundamentales.

La memoria es la capacidad de retener en la mente los acontecimientos pasados y poder recordarlos para usarlos en el futuro. Best (2001) enfatiza el sentido de la memoria como depósito de almacenamiento para mantener o almacenar cosas. Para él, la memoria está dividida en tres almacenes que son: almacén sensorial, almacén a corto plazo y almacén a largo plazo. Se tuvo en cuenta en este estudio el almacén a corto plazo, llamado también por Schunk (1997) memoria a corto plazo y memoria de trabajo. En el almacén a corto plazo el material está organizado acústicamente, la duración del material no elaborado es de unos 30 segundos y el que no es repasado se desvanece, y los códigos almacenados pueden ser trasferidos al "almacén de largo plazo" (Best, 2001).

El lenguaje es el medio por el cual nos comunicamos, según Santrock (2001) “El lenguaje es una forma de comunicación, ya sea oral, escrita o mediante señas, que se basa en un sistema de símbolos" (p. 70).

El lenguaje, en términos funcionales de comprensión verbal, implica el desarrollo sintáctico y semántico. La sintaxis estudia la manera como las palabras se combinan para formar frases o enunciados aceptables y la semántica se refiere al significado de las palabras y las oraciones (Santrock, 2001). En cuanto a la sintaxis u orden de las palabras en frases u oraciones; Woolfolk (1999) afirma que los niños pronto dominan los fundamentos del orden de las palabras, pero necesitan mayor tiempo para dominar las formas más complicadas como la voz pasiva. Alcanzan a comprender y luego usar las estructuras gramaticales complejas como artículos, adjetivos y conjunciones. Respecto del desarrollo semántico (significado de las palabras y las oraciones) afirma que entre los dos y los seis años el niño promedio aprende de seis a diez palabras al día, lo que significa que para los seis años tiene un léxico de 8000 a 14000 palabras. Entre los nueve y once años agrega 5000 nuevas palabras a su repertorio.

El pensamiento es la capacidad de analizar, comparar, evaluar, ordenar, clasificar, hacer inferencias, hacer juicios, aplicar adecuadamente los conocimientos, trascender la información recibida, generar nuevas ideas y resolver problemas.

Según Santrok (2001) "El pensamiento significa manipular y transformar la información en la memoria. Esto a menudo se hace para formar conceptos, razonar, pensar críticamente y resolver problemas" (p. 75).

Según Robledo, R. (2010), los alumnos, dependiendo del curso en el que estén y la especialidad que eligen, presentan unos intereses y motivaciones diferentes hacia el aprendizaje, así como unos estilos de pensamiento específicos y delimitados que se deben considerar a la hora de trabajar con ellos de manera efectiva.

Investigaciones como la que se propone, contribuyen para que las instituciones tomen concien- 
cia de la importancia de un seguimiento cercano de los estudiantes de manera que, desde el inicio del periodo académico, se identifiquen las dificultades y se pueda actuar sobre ellas para evitar la deserción, la repitencia, el impacto psicológico de un desempeño deficiente en la autoestima, autoeficacia y autoimagen, las manifestaciones de ansiedad y el temor que les produce enfrentarse a tales dificultades.

La pregunta de investigación es la siguiente: ¿Qué características presentan los procesos cognitivos de memoria, lenguaje y pensamiento, en estudiantes con bajo y alto rendimiento académico que cursan los estudios escolares entre primero y quinto grado en una institución educativa de la ciudad de Bogotá?

El objetivo principal de la investigación fue caracterizar los procesos cognitivos de memoria, lenguaje y pensamiento, en una muestra de estudiantes que cursan los estudios escolares entre primero y quinto grado en una institución educativa de la ciudad de Bogotá. Los objetivos específicos son, en primer lugar, describir el estado funcional de los procesos cognitivos de memoria, lenguaje y pensamiento en cada uno de los grupos; $y$, en segundo lugar, establecer comparativamente las diferencias y semejanzas en el funcionamiento de los procesos cognitivos de memoria, lenguaje y pensamiento, entre los estudiantes participantes.

\section{Variables}

\section{Rendimiento académico}

Nivel de conocimientos adquiridos en determinadas materias o áreas educativas comparando la edad y el grado escolar. En él influyen muchos factores como el nivel socioeconómico, los programas de estudio, metodologías usadas en la enseñanza, nivel de pensamiento de los alumnos (Benitez, Giménez \& Osicka, 2000), los factores familiares y personales (Ivanovich \& Ivanovich, 1988), el grupo de pares, el contexto educativo y las relaciones interpersonales.
En esta investigación, para determinar quienes tienen bajo, medio, alto y superior rendimiento académico, se tuvo en cuenta los informes académicos respectivos cuyas calificaciones oscilan entre bajo (10-67), básico o medio (68-83), alto (84-94) y superior (95-100).

Esta variable tiene una naturaleza categórica, bajo, medio, alto y superior rendimiento académico. Escala de medición: nominal.

\section{Memoria}

Capacidad de retener los acontecimientos pasados y poder recordarlos para usarlos más tarde. Instrumento de medición de la categoría: WISC - IV. Se utilizaron las subpruebas de retención de dígitos y sucesión de números y letras, que son las que miden la memoria de trabajo. Escala de medición: Intervalo. En el lenguaje de variables esta categoría constituye una variable predictora de naturaleza continua.

\section{Lenguaje}

Medio por el cual nos comunicamos. Segun Santrock (2001) "El lenguaje es una forma de comunicación, ya sea oral, escrita o mediante señas, que se basa en un sistema de símbolos" (p. 70).

El lenguaje, en términos funcionales de comprensión verbal, implica el desarrollo sintáctico y semántico.

Instrumento de medición de la categoría: WISCIV. Se utilizaron las subpruebas de semejanzas, vocabulario y comprensión, que son las que miden la comprensión verbal. Escala de medición: Intervalo. En el lenguaje de variables esta categoría constituye una variable predictora de naturaleza continua.

\section{Pensamiento}

Según Santrok (2001) “El pensamiento significa manipular y transformar la información en la memoria. Esto a menudo se hace para formar con- 
ceptos, razonar, pensar críticamente y resolver problemas" (p. 75).

El pensamiento es la capacidad de analizar, comparar, evaluar, ordenar, clasificar, hacer inferencias, hacer juicios, aplicar adecuadamente los conocimientos, trascender la información recibida, generar nuevas ideas y resolver problemas.

Instrumento de medición de la categoría: WISC IV. Se evaluó mediante las subpruebas de diseño con cubos, conceptos con dibujos y matrices, que son las que miden el razonamiento. Escala de medición: Intervalo. En el lenguaje de variables esta categoría constituye una variable predictora de naturaleza continua.

\section{Método}

\section{Tipo de estudio y diseño}

El presente estudio empírico analítico presenta dos características que lo definen, ser descriptivo y comparativo. La investigación descriptiva, afirma Hernández Sampieri "busca especificar propiedades, características y rasgos importantes de cualquier fenómeno que se analice. Describe tendencias de un grupo o población” (2008, p. 103).

Igualmente, es comparativo entre variables categóricas y continuas, lo que permite aplicar ANOVAS, a partir de los datos suministrados por las diez subpruebas de la "Escala Wechsler de inteligencia para niños (WISC - IV)" a los cuatro grupos intencionales con bajo, medio, alto y superior rendimiento académico, con el fin de establecer diferencias y semejanzas respecto de los procesos cognitivos de memoria, lenguaje y pensamiento.

\section{Participantes}

Cuatro grupos intencionales de estudiantes con bajo, medio, alto y superior rendimiento académico, basados en el promedio de notas de los informes académicos respectivos. Se tomaron estudiantes con edades comprendidas entre seis y once años, de los grados primero a quinto de educación básica primaria, de los cuales doce pertenecen al género masculino y ocho al género femenino.

\section{Instrumento}

Para evaluar la capacidad intelectual de los veinte participantes se aplicaron diez de las quince subpruebas de la Escala Wechsler de inteligencia para niños (WISC - IV), cuyo ámbito de acción es para niños, niñas y adolescentes comprendidos entre seis años cero meses y dieciséis años once meses.

Para desarrollar la investigación, se tuvieron en cuenta las subpruebas que más contribuyen al conocimiento de los niños y adolescentes en los tres aspectos a estudiar, así:

Para evaluar la memoria a corto plazo o memoria de trabajo, las subpruebas de retención de dígitos y sucesión de números y letras; para medir el lenguaje: semejanzas, vocabulario y comprensión; y para medir pensamiento: diseño con cubos, conceptos con dibujos y matrices.

Además de las subpruebas para medir memoria de trabajo, comprensión verbal y razonamiento perceptual, se aplicó a los estudiantes las subpruebas que miden la velocidad de procesamiento que son: subprueba de claves y subprueba de búsqueda de símbolos. Al aplicar todas las diez subpruebas antes referidas, se pudo determinar la capacidad intelectual de cada estudiante.

\section{Procedimiento}

Para el desarrollo de la presente investigación se tuvieron en cuentas las siguientes fases:

Fase 1. Ajuste de un modelo teórico del rendimiento académico en el que los procesos de memoria, lenguaje y pensamiento juegan un papel importante.

Fase 2. Revisión de los informes académicos correspondientes para determinar el rendimiento 
académico en estudiantes con bajo, medio, alto y superior rendimiento académico.

Fase 3. Aplicación de las subpruebas elegidas de WISC - IV.

Fase 4. Calificación de las subpruebas.

Fase 5. Sistematización y codificación de resultados mediante SPSS. Se hallaron las medidas de tendencia central, media, mediana, moda y desviación. A través de ANOVA y DHS (Diferencia Honestamente Significativa) de Tukey, se establecieron las diferencias y semejanzas entre los grupos participantes.

Fase 6. Análisis comparativo de las diferencias y semejanzas más relevantes en el funcionamiento de los procesos cognitivos de memoria, lenguaje y pensamiento, entre los grupos de estudiantes con bajo, medio, alto y superior rendimiento académico.

Fase 7. Discusión.

\section{Aspectos éticos}

La investigación se ciñó a la ley 1090 de 2006, por la cual se reglamenta el ejercicio de la profesión de Psicología en Colombia, se dicta el código deontológico y bioético y otras disposiciones. El artículo 2, numeral 3 sobre estándares morales y legales; el numeral 5 sobre la confidencialidad, y el numeral 6 sobre el bienestar del usuario; también el artículo 50 sobre los principios éticos de respeto y dignidad lo mismo que salvaguardar el bienestar y los derechos de los participantes; el artículo $36 \mathrm{i}$.

No se practicaron intervenciones sin consentimiento autorizado del usuario, o en casos de menores de edad o dependientes, del consentimiento del acudiente y el artículo 52 que dice que en los casos de menores de edad y personas incapacitadas, el consentimiento respectivo deberá firmarlo el representante legal del participante.

\section{Resultados}

En esta investigación se clasificaron a los estudiantes en los diferentes grupos teniendo en cuenta los informes académicos respectivos, cuyas calificaciones oscilan entre 10 y 67 (bajo), 68 y 83 (medio), 84 y 94 (alto) y entre 95 y 100 (superior).

Tabla 1.

Calificaciones promedio y concepto de rendimiento académico

\begin{tabular}{ccc}
\hline$N^{\circ}$ alumno & $\begin{array}{c}\text { Calificación } \\
\text { (promedio) }\end{array}$ & concepto \\
\hline 1 & 99 & Superior \\
2 & 98 & Superior \\
3 & 68 & Medio \\
4 & 38 & Bajo \\
5 & 93 & Alto \\
6 & 61 & Bajo \\
7 & 90 & Alto \\
8 & 70 & Medio \\
9 & 68 & Medio \\
10 & 50 & Bajo \\
11 & 57 & Bajo \\
12 & 61 & Bajo \\
13 & 90 & Alto \\
14 & 93 & Alto \\
15 & 89 & Alto \\
16 & 69 & Medio \\
17 & 91 & Alto \\
18 & 90 & Alto \\
19 & 87 & Alto \\
20 & 70 & Medio \\
\hline
\end{tabular}

La Tabla 1 presenta las calificaciones promedio y el concepto de rendimiento académico según el sistema de calificaciones de la institución educativa, de donde proceden los estudiantes con quienes se desarrolló la investigación. El menor rendimiento académico lo presenta un estudiante que tiene nota promedio de 38 (bajo) y el mayor rendimiento lo presenta otro estudiante que tiene nota promedio de 99 (superior). Cinco estudiantes presentan bajo rendimiento académico; cinco, rendimiento académico básico; ocho rendimiento académico alto y dos rendimiento académico superior. 
Tabla 2.

Estadísticos descriptivos de las variables calificaciones, memoria, lenguaje, pensamiento, procesamiento y cociente intelectual

\begin{tabular}{lcccccc}
\hline & Calificaciones & $\begin{array}{c}\text { Memoria } \\
\text { de trabajo }\end{array}$ & $\begin{array}{c}\text { Comprensión } \\
\text { verbal }\end{array}$ & $\begin{array}{c}\text { Razonamiento } \\
\text { perceptual }\end{array}$ & $\begin{array}{c}\text { Velocidad de } \\
\text { procesamiento }\end{array}$ & $\begin{array}{c}\text { Cociente } \\
\text { intelectual }\end{array}$ \\
\hline N Válidos & 20 & 20 & 20 & 20 & 20 & 20 \\
Perdidos & 0 & 0 & 0 & 0 & 0 & 0 \\
Media & 76,60 & 96,45 & 101,40 & 97,75 & 98,70 & 98,95 \\
Mediana & 78,50 & 100,50 & 103,50 & 100,00 & 100,00 & 106,50 \\
Moda & 90 & 91 & 110 & 104 & $88(a)$ & $81(a)$ \\
Desv. típ. & 17,572 & 14,745 & 19,250 & 13,626 & 17,128 & 18,066 \\
Varianza & 308,779 & 217,418 & 370,568 & 185,671 & 293,379 & 326,366 \\
\hline
\end{tabular}

\section{Descriptivo de variables}

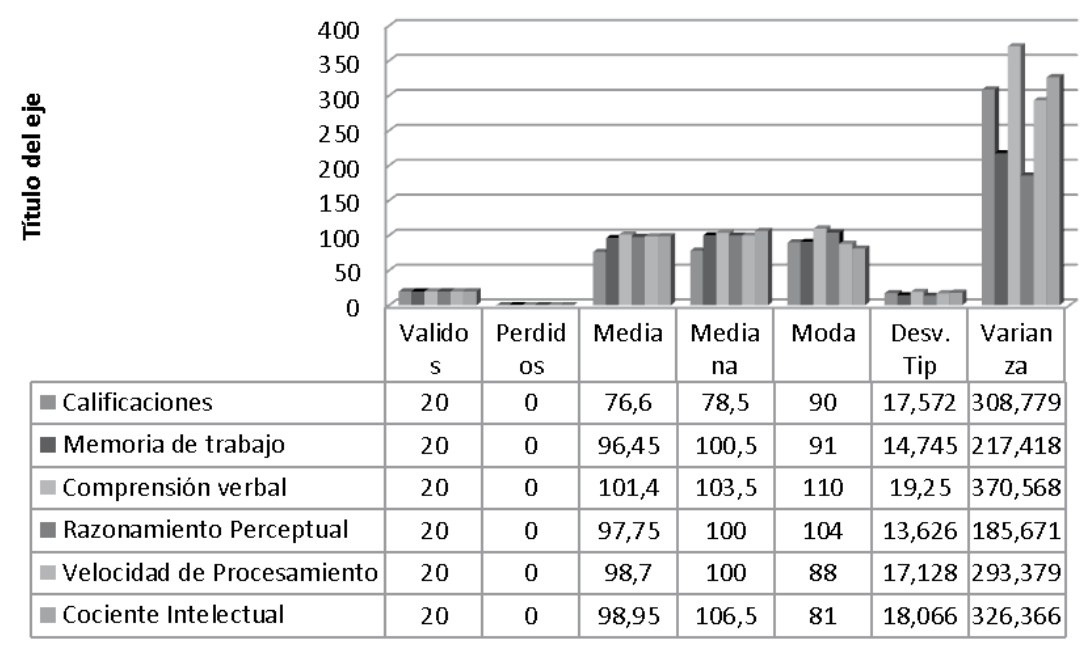

Figura 1. Estadísticos descriptivos de las variables calificaciones, memoria, lenguaje, pensamiento, procesamiento y cociente intelectual.

La Tabla 2 y Figura 1 presentan un compendio de las medidas de tendencia central con respecto a calificaciones, memoria de trabajo, comprensión verbal, razonamiento perceptual, velocidad de procesamiento y cociente intelectual.

Los resultados muestran que el promedio de las calificaciones de los estudiantes, en una escala de 1 a 100 , es de 76,6 . El $50 \%$ de los estudiantes están por encima del valor 78,5 correspondiente a la mediana y el restante $50 \%$ se sitúa por debajo de este valor. La puntuación que se presenta con mayor frecuencia es 90 (Moda). De la media de 76,6 se observa una desviación típica de 17,5. En síntesis y de acuerdo con los valores obtenidos, las puntuaciones de las calificaciones tienden a ubicarse en rendimiento académico medio o básico.

De acuerdo con las descripciones cualitativas de las puntuaciones compuestas presentadas en el manual técnico WISC- IV, de Wechsler, D. (2007, 
p. 99), en orden descendente el promedio (media) es el siguiente: comprensión verbal 101,7; razonamiento perceptual 97,7; memoria de trabajo 96,4.

En CV el $50 \%$ de los estudiantes está por encima y el otro $50 \%$ está por debajo del valor 103,5; en RP el $50 \%$ por encima y el $50 \%$ por debajo del valor 100,0 ; en MT el $50 \%$ por encima y el $50 \%$ por debajo de la mediana 100,5.
La puntuación que se presenta con mayor frecuencia (moda) en CV es 110; en RP 104; y en MT 91.

Se observa por lo tanto que el orden de influencia en el rendimiento académico es el siguiente: en primer lugar la CV, en segundo lugar el RP y en tercer lugar la MT. Con un promedio de 101,7; 97,7 y 96.4 respectivamente.

Tabla 3.

ANOVA de un factor. Descriptivos

\begin{tabular}{|c|c|c|c|c|c|c|c|c|c|}
\hline & & \multirow{2}{*}{$\begin{array}{c}\mathbf{N} \\
\begin{array}{c}\text { Límite } \\
\text { inferior }\end{array}\end{array}$} & \multirow{2}{*}{$\begin{array}{c}\text { Media } \\
\begin{array}{c}\text { Límite } \\
\text { superior }\end{array}\end{array}$} & \multirow{2}{*}{$\begin{array}{c}\begin{array}{c}\text { Des- } \\
\text { viación } \\
\text { típica }\end{array} \\
\begin{array}{c}\text { Límite } \\
\text { inferior }\end{array}\end{array}$} & \multirow{2}{*}{$\begin{array}{c}\begin{array}{c}\text { Error } \\
\text { típico }\end{array} \\
\begin{array}{c}\text { Límite } \\
\text { superior }\end{array}\end{array}$} & \multicolumn{2}{|c|}{$\begin{array}{c}\text { Intervalo confian- } \\
\text { za } 95 \%\end{array}$} & \multirow{2}{*}{$\begin{array}{c}\text { Mínimo } \\
\begin{array}{c}\text { Límite } \\
\text { inferior }\end{array}\end{array}$} & \multirow{2}{*}{$\begin{array}{c}\text { Máximo } \\
\begin{array}{c}\text { Límite } \\
\text { superior }\end{array}\end{array}$} \\
\hline & & & & & & $\begin{array}{l}\text { Límite } \\
\text { inferior }\end{array}$ & $\begin{array}{l}\text { Límite } \\
\text { superior }\end{array}$ & & \\
\hline \multirow[t]{5}{*}{ Calificaciones } & bajo & 5 & 53,40 & 9,711 & 4,343 & 41,34 & 65,46 & 38 & 61 \\
\hline & medio & 5 & 69,00 & 1,000 & ,447 & 67,76 & 70,24 & 68 & 70 \\
\hline & alto & 8 & 90,38 & 1,996 & ,706 & 88,71 & 92,04 & 87 & 93 \\
\hline & superior & 2 & 98,50 & ,707 &, 500 & 92,15 & 104,85 & 98 & 99 \\
\hline & Total & 20 & 76,60 & 17,572 & 3,929 & 68,38 & 84,82 & 38 & 99 \\
\hline \multirow[t]{5}{*}{$\begin{array}{l}\text { Memoria de } \\
\text { trabajo }\end{array}$} & bajo & 5 & 87,00 & 11,467 & 5,128 & 72,76 & 101,24 & 74 & 102 \\
\hline & medio & 5 & 89,40 & 19,034 & 8,512 & 65,77 & 113,03 & 59 & 104 \\
\hline & alto & 8 & 104,00 & 9,381 & 3,317 & 96,16 & 111,84 & 91 & 116 \\
\hline & superior & 2 & 107,50 & 12,021 & 8,500 &,- 50 & 215,50 & 99 & 116 \\
\hline & Total & 20 & 96,45 & 14,745 & 3,297 & 89,55 & 103,35 & 59 & 116 \\
\hline \multirow[t]{5}{*}{$\begin{array}{l}\text { Comprensión } \\
\text { verbal }\end{array}$} & bajo & 5 & 82,00 & 16,062 & 7,183 & 62,06 & 101,94 & 55 & 96 \\
\hline & medio & 5 & 99,00 & 22,260 & 9,955 & 71,36 & 126,64 & 71 & 130 \\
\hline & alto & 8 & 112,88 & 11,432 & 4,042 & 103,32 & 122,43 & 98 & 130 \\
\hline & superior & 2 & 110,00 & ,000 & ,000 & 110,00 & 110,00 & 110 & 110 \\
\hline & Total & 20 & 101,40 & 19,250 & 4,304 & 92,39 & 110,41 & 55 & 130 \\
\hline \multirow[t]{5}{*}{$\begin{array}{l}\text { Razonamiento } \\
\text { perceptual }\end{array}$} & bajo & 5 & 84,40 & 9,762 & 4,366 & 72,28 & 96,52 & 73 & 94 \\
\hline & medio & 5 & 96,00 & 16,628 & 7,436 & 75,35 & 116,65 & 75 & 117 \\
\hline & alto & 8 & 105,88 & 8,855 & 3,131 & 98,47 & 113,28 & 96 & 123 \\
\hline & superior & 2 & 103,00 & 1,414 & 1,000 & 90,29 & 115,71 & 102 & 104 \\
\hline & Total & 20 & 97,75 & 13,626 & 3,047 & 91,37 & 104,13 & 73 & 123 \\
\hline \multirow[t]{3}{*}{$\begin{array}{l}\text { Velocidad de } \\
\text { procesamiento }\end{array}$} & bajo & 5 & 82,40 & 8,173 & 3,655 & 72,25 & 92,55 & 70 & 88 \\
\hline & medio & 5 & 89,20 & 16,392 & 7,331 & 68,85 & 109,55 & 78 & 118 \\
\hline & alto & 8 & 108,63 & 7,763 & 2,745 & 102,13 & 115,12 & 97 & 118 \\
\hline
\end{tabular}




\begin{tabular}{|c|c|c|c|c|c|c|c|c|c|}
\hline & & \multirow{2}{*}{$\begin{array}{c}\mathrm{N} \\
\begin{array}{c}\text { Límite } \\
\text { inferior }\end{array}\end{array}$} & \multirow{2}{*}{$\begin{array}{c}\text { Media } \\
\begin{array}{c}\text { Límite } \\
\text { superior }\end{array}\end{array}$} & \multirow{2}{*}{$\begin{array}{c}\begin{array}{c}\text { Des- } \\
\text { viación } \\
\text { típica }\end{array} \\
\begin{array}{c}\text { Límite } \\
\text { inferior }\end{array}\end{array}$} & \multirow{2}{*}{$\begin{array}{c}\begin{array}{c}\text { Error } \\
\text { típico }\end{array} \\
\begin{array}{c}\text { Límite } \\
\text { superior }\end{array}\end{array}$} & \multicolumn{2}{|c|}{$\begin{array}{l}\text { Intervalo confian- } \\
\text { za } 95 \%\end{array}$} & \multirow{2}{*}{$\begin{array}{c}\text { Mínimo } \\
\begin{array}{c}\text { Límite } \\
\text { inferior }\end{array}\end{array}$} & \multirow{2}{*}{$\begin{array}{c}\text { Máximo } \\
\begin{array}{c}\text { Límite } \\
\text { superior }\end{array}\end{array}$} \\
\hline & & & & & & $\begin{array}{l}\text { Límite } \\
\text { inferior }\end{array}$ & $\begin{array}{l}\text { Límite } \\
\text { superior }\end{array}$ & & \\
\hline & superior & 2 & 123,50 & 3,536 & 2,500 & 91,73 & 155,27 & 121 & 126 \\
\hline & Total & 20 & 98,70 & 17,128 & 3,830 & 90,68 & 106,72 & 70 & 126 \\
\hline \multirow[t]{5}{*}{$\begin{array}{l}\text { Cociente } \\
\text { intelectual }\end{array}$} & bajo & 5 & 79,40 & 11,127 & 4,976 & 65,58 & 93,22 & 60 & 88 \\
\hline & medio & 5 & 92,80 & 21,052 & 9,415 & 66,66 & 118,94 & 65 & 119 \\
\hline & alto & 8 & 111,25 & 4,803 & 1,698 & 107,23 & 115,27 & 105 & 119 \\
\hline & superior & 2 & 114,00 & 2,828 & 2,000 & 88,59 & 139,41 & 112 & 116 \\
\hline & Total & 20 & 98,95 & 18,066 & 4,040 & 90,50 & 107,40 & 60 & 119 \\
\hline
\end{tabular}

La Tabla 3 presenta las relaciones que se dan entre calificaciones, memoria de trabajo, comprensión verbal, razonamiento perceptual, velocidad de procesamiento y cociente intelectual, con el rendimiento académico bajo, medio, alto y superior.

De los 20 estudiantes, cinco presentan bajo rendimiento académico; cinco, rendimiento académico medio; ocho, rendimiento académico alto y dos, rendimiento académico superior. Para el análisis de la Tabla 3, y para establecer una comparación global, se realizó una fusión en dos grandes grupos, "bajo rendimiento académico" (los de bajo y medio) y "alto rendimiento académico" (los de alto y superior).

En calificaciones, los estudiantes del grupo con rendimiento académico bajo y medio obtuvieron diferencias significativas respecto a los estudiantes del grupo con alto y superior rendimiento; mientras que los estudiantes del primer grupo alcanzaron un puntaje promedio de 61.2 en sus calificaciones, los del segundo alcanzaron un promedio de 94.4. El promedio total es de 76,6.

En el puntaje de memoria de trabajo, los estudiantes del grupo de bajo y medio rendimiento académico presentan diferencias significativas respecto a los estudiantes del grupo con alto y superior rendimiento; mientras los primeros pre- sentan un puntaje promedio de 88.2 , los segundos presentan un promedio de 105.7. El promedio total es de 96.4

En comprensión verbal, los estudiantes del grupo con bajo y medio rendimiento académico presentan diferencias significativas con respecto al grupo con alto y superior rendimiento; mientras los primeros presentan un puntaje promedio de 90.5 , los segundos presentan un promedio de 111.4. El promedio total es de 101,4.

En razonamiento perceptual, el grupo de estudiantes con rendimiento académico bajo y medio, pues mientras los primeros presentan un puntaje promedio de 87.2 , los segundos un promedio de 104.4. El promedio total es de 97,5 .

En velocidad de procesamiento las diferencias son significativas entre el grupo de estudiantes con bajo y medio rendimiento académico, y el grupo con alto y superior rendimiento; pues los primeros presentan un puntaje promedio de 85.8 , mientras los segundos presentan un promedio de 116.0. Lo que implica una gran diferencia, por lo tanto, se aprecia que la velocidad de procesamiento es fundamental en el rendimiento académico. El puntaje promedio total fue de 98,7.

En cociente intelectual existen diferencias significativas entre el grupo de estudiantes con 
rendimiento académico bajo y medio, y el grupo de estudiantes con alto y superior rendimiento, pues mientras el primero presenta un puntaje promedio de 81.1, el segundo presenta un promedio de 112.6. El promedio total es de 98,9.

En resumen, teniendo en cuenta la división de los grupos en esta investigación:

El puntaje en calificaciones del grupo con bajo rendimiento académico fue de 61.2 y el del grupo con alto rendimiento académico de 94.4. Promedio 76.6.

El puntaje en memoria de trabajo del grupo con bajo rendimiento académico fue de 88.2 y el del grupo con alto rendimiento académico de 105.7. Promedio 96.4.

El puntaje en comprensión verbal del grupo con bajo rendimiento académico fue de $90.5 \mathrm{y}$ el del grupo con alto rendimiento académico de 111.4. Promedio 101.4.

El puntaje de razonamiento perceptual del grupo con bajo rendimiento académico fue de 87.2 y el del grupo con alto rendimiento académico de 104.4. Promedio total 97.7.
El puntaje de velocidad de procesamiento del grupo con bajo rendimiento académico fue de 85.5 y el del grupo con alto rendimiento académico de 116.0. Promedio total 98.7.

El puntaje de cociente intelectual del grupo con bajo rendimiento académico fue de $81.1 \mathrm{y}$ el del grupo con alto rendimiento académico de 112.6. Promedio total 98.9.

Al igual que los resultados arrojados en la Tabla 2, en la Tabla 3 se evidencia que en el rendimiento académico influye en primer lugar la CV 101,4: en segundo lugar el RP 97,7 y en tercer lugar la MT con un puntaje de 96,4 .

Las mayores diferencias entre el grupo de estudiantes con menor y el grupo con mayor rendimiento académico, en los tres aspectos que nos interesan en esta investigación (memoria, lenguaje y pensamiento), se dieron en primer lugar en lenguaje (90.5-111.4 puntos respectivamente), para una diferencia de 20.9 puntos; en segundo lugar en memoria (88.2-105.7 puntos respectivamente) para una diferencia de 17.5 puntos; y por último, en pensamiento (87.2-104.4 puntos respectivamente) para una diferencia de 17.2 puntos.

Tabla 4.

Prueba T: Estadísticos de grupo entre género y calificaciones, memoria de trabajo, comprensión verbal, razonamiento perceptual, velocidad de procesamiento, cociente intelectual

\begin{tabular}{lccccc}
\hline & Género & N & Media & Desviación típ. & Error típ. de la media \\
\hline Calificaciones & masculino & 12 & 76,50 & 15,187 & 4,384 \\
& femenino & 8 & 76,75 & 21,809 & 7,711 \\
Memoria de trabajo & masculino & 12 & 94,00 & 16,553 & 4,778 \\
& femenino & 8 & 100,13 & 11,569 & 4,090 \\
Comprensión verbal & masculino & 12 & 103,83 & 19,366 & 5,591 \\
Razonamiento perceptual & femenino & 8 & 97,75 & 19,776 & 6,992 \\
\multirow{3}{*}{ Velocidad de procesamiento } & masculino & 12 & 100,42 & 13,501 & 3,897 \\
& femenino & 8 & 93,75 & 13,677 & 4,836 \\
cociente intelectual & masculino & 12 & 96,67 & 16,560 & 4,781 \\
& femenino & 8 & 101,75 & 18,645 & 6,592 \\
& masculino & 12 & 99,50 & 18,133 & 5,235 \\
\hline
\end{tabular}


La Tabla 4 presenta una comparación entre calificaciones, memoria de trabajo, comprensión verbal, razonamiento perceptual, velocidad de procesamiento, cociente intelectual y el género masculino y femenino.

Haciendo una comparación general entre los estudiantes de género masculino y género femenino no existen diferencias significativas en el puntaje promedio; sin embargo, cabe destacar que las mayores semejanzas se presentan en las calificaciones y el cociente intelectual y que, en los demás aspectos, se presentan pequeñas diferencias.
Los puntajes promedio en el género masculino son mayores que los del género femenino en: razonamiento perceptual $(100,4$ y 93,7 respectivamente), comprensión verbal (103,8 y 97,7 respectivamente), y cociente intelectual $(99,5$ y 98,1 respectivamente).

Los puntajes promedio en el género femenino son mayores que los del género masculino en: memoria de trabajo $(100,1$ y 94 respectivamente), velocidad de procesamiento (101,7 y 96,6 respectivamente), y calificaciones (76,7 y 76,5 respectivamente).

Tabla 5.

Correlaciones

\begin{tabular}{|c|c|c|c|c|c|c|c|}
\hline & & $\begin{array}{l}\text { Calificacio- } \\
\text { nes }\end{array}$ & $\begin{array}{l}\text { Memoria } \\
\text { de trabajo }\end{array}$ & $\begin{array}{l}\text { Compren- } \\
\text { sión verbal }\end{array}$ & $\begin{array}{c}\text { Razona- } \\
\text { miento } \\
\text { perceptual }\end{array}$ & $\begin{array}{l}\text { Velocidad } \\
\text { de procesa- } \\
\text { miento }\end{array}$ & $\begin{array}{l}\text { Cociente } \\
\text { intelectual }\end{array}$ \\
\hline \multirow[t]{3}{*}{ Calificaciones } & $\begin{array}{l}\text { Correlación } \\
\text { de Pearson }\end{array}$ & 1 &, $577\left(^{* *}\right)$ &, $718\left(^{* *}\right)$ &, $\left.659^{(* *}\right)$ &, $822\left({ }^{* *}\right)$ &, $\left.819^{* *}\right)$ \\
\hline & Sig. (bilateral) & & ,008 & ,000 & ,002 & ,000 & ,000 \\
\hline & N & 20 & 20 & 20 & 20 & 20 & 20 \\
\hline \multirow[t]{3}{*}{$\begin{array}{l}\text { Memoria de } \\
\text { trabajo }\end{array}$} & $\begin{array}{l}\text { Correlación } \\
\text { de Pearson }\end{array}$ &, $577\left(^{* *}\right)$ & 1 &, $661\left(^{* *}\right)$ &, $\left.5611^{*}\right)$ &, $719\left(^{* *}\right)$ &, $824\left(^{* *}\right)$ \\
\hline & Sig. (bilateral) & ,008 & & ,002 & ,010 & ,000 & ,000 \\
\hline & N & 20 & 20 & 20 & 20 & 20 & 20 \\
\hline \multirow[t]{3}{*}{$\begin{array}{l}\text { Comprensión } \\
\text { verbal }\end{array}$} & $\begin{array}{l}\text { Correlación } \\
\text { de Pearson }\end{array}$ &, $718\left(^{* *}\right)$ &, $661\left({ }^{* *}\right)$ & 1 & ,652(**) &, $\left.708{ }^{* *}\right)$ &, $908\left(^{* *}\right)$ \\
\hline & Sig. (bilateral) &, 000 &, 002 & & ,002 &, 000 &, 000 \\
\hline & N & 20 & 20 & 20 & 20 & 20 & 20 \\
\hline \multirow[t]{3}{*}{$\begin{array}{l}\text { Razonamiento } \\
\text { perceptual }\end{array}$} & $\begin{array}{l}\text { Correlación } \\
\text { de Pearson }\end{array}$ & ,659(**) &, $561\left(^{*}\right)$ & ,652( $\left.{ }^{* *}\right)$ & 1 & ,616(**) &, $818\left(^{* *}\right)$ \\
\hline & Sig. (bilateral) &, 002 &, 010 &, 002 & & ,004 &, 000 \\
\hline & $\mathrm{N}$ & 20 & 20 & 20 & 20 & 20 & 20 \\
\hline \multirow[t]{3}{*}{$\begin{array}{l}\text { Velocidad de } \\
\text { procesamiento }\end{array}$} & $\begin{array}{l}\text { Correlación } \\
\text { de Pearson }\end{array}$ &, $822\left(^{* *}\right)$ &, $719\left(^{* *}\right)$ &, $708\left(^{* *}\right)$ & ,616(**) & 1 &, $878\left(^{* *}\right)$ \\
\hline & Sig. (bilateral) & ,000 & ,000 &, 000 &, 004 & &, 000 \\
\hline & $\mathrm{N}$ & 20 & 20 & 20 & 20 & 20 & 20 \\
\hline \multirow[t]{3}{*}{$\begin{array}{l}\text { Cociente } \\
\text { intelectual }\end{array}$} & $\begin{array}{l}\text { Correlación } \\
\text { de Pearson }\end{array}$ &, $819\left(^{* *}\right)$ &, $824\left(^{* *}\right)$ &, $908\left(^{* *}\right)$ &, $818\left(^{* *}\right)$ &, $878\left(^{* *}\right)$ & 1 \\
\hline & Sig. (bilateral) &, 000 & ,000 &, 000 & ,000 &, 000 & \\
\hline & $\mathrm{N}$ & 20 & 20 & 20 & 20 & 20 & 20 \\
\hline
\end{tabular}

** La correlación es significativa al nivel 0,01 (bilateral).

* La correlación es significante al nivel 0,05 (bilateral). 


\title{
CORRELACIONES
}

\author{
CORRELACIONES
}

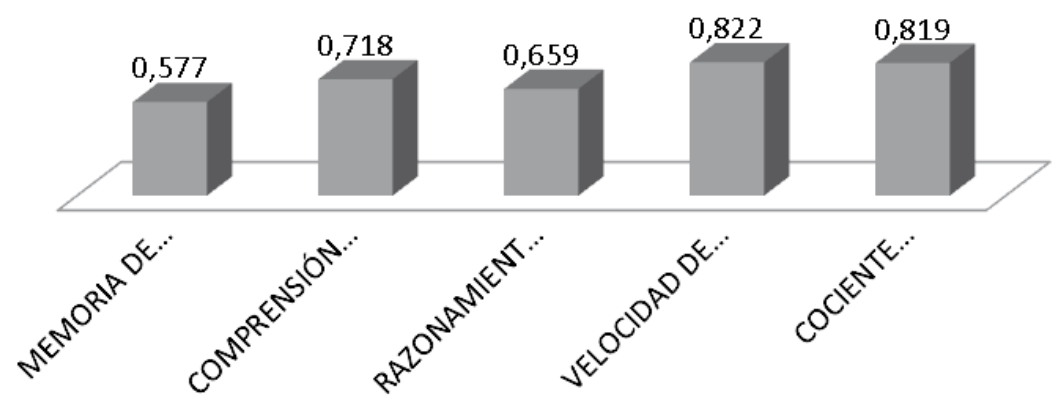

Figura. Correlaciones entre rendimiento académico, memoria, lenguaje, pensamiento, velocidad de procesamiento y cociente intelectual.

La Tabla 5 y la Figura 2 presentan las correlaciones que existen entre calificaciones, memoria de trabajo, comprensión verbal, razonamiento perceptual, velocidad de procesamiento, cociente intelectual.

Se analizaron las correlaciones más significativas y las menos significativas entre calificaciones, memoria de trabajo, comprensión verbal y razonamiento perceptual, que son las variables que más interesan en el presente estudio.

La correlación de las calificaciones es significativa con comprensión verbal $(, 718)$ y mínima con memoria de trabajo $(, 577)$; la correlación de memoria de trabajo es significativa con comprensión verbal $(, 661)$ y mínima con razonamiento perceptual (,561); la correlación de comprensión verbal el significativa con las calificaciones $(, 718)$ y mínima con razonamiento perceptual $(, 652)$; la correlación del razonamiento perceptual es significativo con las calificaciones $(, 659)$ y mínima con memoria de trabajo $(, 561)$.

El aspecto que más influye en las calificaciones (que son las que determinan el rendimiento académico) es la comprensión verbal con una correlación de ,718; le sigue el razonamiento perceptual con una correlación de ,659 y el que menos influye es la memoria de trabajo con una correlación de ,577.

De todos los datos anteriores se concluye que existen diferencias significativas al comparar las variables de memoria, lenguaje y pensamiento, mediante las pruebas de diferencias de medias, lo que indica que los estudiantes con bajo rendimiento académico presentan bajos puntajes en las variables de memoria, lenguaje y pensamiento; mientras que los estudiantes con rendimiento académico superior presentan altos puntajes en las variables de memoria, lenguaje y pensamiento. Dicho en otras palabras, existe una relación término a término, entre los estudiantes con bajo, medio, alto y superior rendimiento académico y las pruebas de memoria, lenguaje y pensamiento ofrecidas por el WISC - IV. En su orden, la variable que más influye en el rendimiento académico es el lenguaje, le sigue el pensamiento, y por último, la memoria de trabajo.

\section{Discusión}

El objetivo de la investigación se centró en caracterizar los procesos cognitivos de memoria, lenguaje y pensamiento en dos muestras de estudiantes con bajo, medio, alto y superior rendimiento académico, teniendo en cuenta la escala 
nominativa de la respectiva institución educativa, quienes cursan los estudios escolares entre primero y quinto grado en una institución educativa de la ciudad de Bogotá-Colombia.

Para determinar el rendimiento académico se tuvo en cuenta el sistema de calificaciones: bajo (10-67), medio (68-83), alto (84-94) y superior (95$100)$, que presenta la institución educativa a la que pertenecen los estudiantes participantes en esta investigación.

Se abordó el presente estudio desde un método descriptivo y comparativo. Descriptivo pues busca especificar propiedades, características y rasgos importantes de la memoria, el lenguaje y el pensamiento en dos grupos de estudiantes uno con bajo y otro con alto rendimiento académico. Retomando a Hernandez Sampieri (2008), "describir tendencias de un grupo o población". Es comparativo porque se establecieron diferencias y semejanzas en los procesos cognitivos de memoria, lenguaje y pensamiento entre el grupo de estudiantes con bajo y el grupo de estudiantes con alto rendimiento académico mediante la aplicación de 10 subpruebas de la escala Wechsler para niños (WISC -IV).

El menor rendimiento académico en la escala de 1 a 100 lo presenta un estudiante cuya nota promedio es 38 que corresponde a bajo y el mayor un estudiante cuya nota promedio es de 99 y que corresponde a rendimiento académico superior. De los 20 estudiantes, cinco presentan bajo rendimiento, cinco básico o medio, ocho alto y dos superior rendimiento académico. El promedio de calificaciones es de 76,6 que en el sistema de calificaciones antes referido corresponde a rendimiento académico medio o básico.

Para determinar los procesos cognitivos de memoria, lenguaje y pensamiento se tuvo en cuenta el manual técnico del WISC - IV, de Wechsler (2007, p. 99), cuyas puntuaciones compuestas son: extremadamente bajo (69 y menor), limítrofe (70-79), promedio bajo (80-89), promedio (90109), promedio alto (110-119), superior (120-129) y muy superior (130 y mayor).
En orden descendente el puntaje promedio de los procesos cognitivos en los estudiantes que participaron en la investigación es el siguiente: comprensión verbal 101,4; razonamiento perceptual 97,7 y memoria de trabajo 96.4. Como puede apreciarse, de estos tres aspectos, el que más influye en el rendimiento académico es la comprensión verbal, le sigue el razonamiento perceptual y en menor grado la memoria de trabajo.

Pero en la Tabla 5 se puede apreciar que lo que más influye en las calificaciones (que son las que determinan el rendimiento académico) es la comprensión verbal con una correlación de ,718 seguida por el razonamiento perceptual con una correlación de ,659 y por último la memoria de trabajo con una correlación de ,577. Sigue primando el rendimiento académico la comprensión verbal, pasando al segundo lugar el razonamiento perceptual y al último, la memoria de trabajo.

De los tres procesos cognitivos estudiados en esta investigación se evidencia que la comprensión verbal con un puntaje promedio de 101,4 es la que más influye en el rendimiento académico; le sigue el razonamiento perceptual con un puntaje promedio de 97.7 y en último lugar la memoria de trabajo cuyo puntaje promedio es de 96,4 en los estudiantes a quienes se les aplicó la prueba WISC - IV.

Queda claro la importancia de la memoria, el lenguaje y el pensamiento en el rendimiento académico pues se da una relación término a término entre los estudiantes con bajo y alto rendimiento; mientras que los estudiantes con el menor rendimiento académico presentan bajos puntajes en memoria, lenguaje y pensamiento, los de mayor rendimiento presentan altos puntajes en estos mismos aspectos.

Esta idea es corroborada por Betancourt \& González (2008) quienes afirman: "tradicionalmente se ha señalado la atención, la memoria, el pensamiento y el lenguaje como procesos cuyo insuficiente desarrollo provoca déficits en el aprendizaje" y por ende en el rendimiento académico (p. 31). A lo que añaden Siegel \& Ryan (1989) y Swanson (2000), "cuando se presentan déficits en 
la memoria de trabajo también se presentan problemas en la lectura".

Gómez et ál. (2007) en un estudio para analizar las características fonológicas de la conciencia fonológica y el comportamiento verbal, concluyeron que los niños con dificultades de aprendizaje presentan problemas en la segmentación fonológica, en la comprensión verbal y en las habilidades narrativas, y Das afirma: "el lenguaje es crucial para el desarrollo cognitivo pues proporciona el medio para expresar ideas, plantear preguntas, las categorías y los conceptos para el pensamiento y los vínculos entre el pasado y el futuro" (1995).

En lo que se refiere a la memoria, Castillo-Parra, Gómez y Ostrosky-Solis (2009), en una investigación sobre los efectos de la memoria sobre el rendimiento encontraron que entre mayor es la capacidad de la memoria, el rendimiento académico es mejor y que los alumnos de alto rendimiento académico muestran una mayor capacidad de memoria en comparación al grupo de bajo aprovechamiento escolar (p. 50). Esta misma idea ya había sido corroborada por Aronen et ál. (2005) quienes afirman que los niños entre tres y trece, años con una buena capacidad de memoria cuentan con mejor desempeño académico que aquellos que presentan bajo nivel de memoria y que la evaluación de la capacidad de memoria es sensible para detectar y diferenciar a los niños con un rendimiento académico alto o bajo.

En lo referente al pensamiento, Benites, Giménez \& Osicka (2000) plantean que, entre otros factores, el nivel de pensamiento de los estudiantes influye significativamente en su rendimiento académico.

Algunos alcances de esta investigación son:

Se pudo establecer una relación entre los procesos de memoria, lenguaje y pensamiento con el rendimiento académico, es decir, estas variables cognitivas parecen incidir de una forma significativa en el bajo y alto rendimiento académico. Esto abre una posibilidad de investigación hacia el fu- turo con el fin de establecer el valor que tienen estas variables en la varianza de los resultados.

Se podrían hacer en el futuro investigaciones con poblaciones más grandes que permitan hacer pruebas de regresión que permitan ver una relación un poco más estrecha entre lo cognitivo y el rendimiento académico. Las pruebas de regresión permitirían establecer relaciones mucho más fuertes que nos expliquen el valor de estas variables en la varianza de los resultados.

Con una muestra más grande se podrían realizar estudios de tipo factorial para ver si los procesos cognitivos de memoria, lenguaje y pensamiento se agrupan en uno solo y mirar si en relación con otras variables la variable cognitiva pesa más. Pero estos estudios serían con universos más grandes y con pruebas estadísticas mucho más sofisticadas.

Se podría establecer una relación entre cognición y rendimiento académico manipulando la variable cognición, es decir, haciendo investigaciones experimentales o semi-experimentales donde al manipular las variables de memoria, lenguaje y pensamiento se deberían ver efectos en el rendimiento académico.

Pero esto hace parte de otra investigación que obviamente se inscribe en el campo de la psicología educativa y que implicaría procesos novedosos de enseñanza aprendizaje, que se centrarán en los procesos cognitivos y ver, después de esa manipulación, como el rendimiento académico debería mejorar.

El estudio realizado con los 20 estudiantes logra establecer que sí existe una relación muy significativa entre los procesos de memoria, lenguaje y pensamiento y el bajo o alto rendimiento académico, de donde surgen las siguientes preguntas:

¿Cuál es el peso, el valor, la influencia de este tipo de variables en el rendimiento académico en un marco mucho más complejo de explicación? ¿De qué manera habría que desarrollar los procesos cognitivos de memoria, lenguaje y pensamiento 
de forma que incidan mucho más directamente en el rendimiento académico?

\section{Referencias}

Aronen, E., Vuontela, V., Steenari, M., Salmi, J., \& Carlson, S. (2005). Working memory, psychiatric symptoms, and academic performance at school. Neurobiology of Learning and Memory, 83, 33-42.

Benítez, M. Giménez, M. \& Osicka, R. (2000). Las asignaturas pendientes y el rendimiento académico: ¿existe alguna relación? Recuperado de: http//fai.unne.edu. ar/links/LAS\%2...20EL\%20RENDIMIENTO\%20 ACADEMICO.htm

Best, J. (2001). Psicología del aprendizaje. Madrid: International Thomson.

Betancourt, J. \& González, A. (2003). Dificultades en el aprendizaje y trastornos emocionales y de la conducta. La Habana: Pueblo y Educación.

Cascón, I. (2000). Análisis de las calificaciones escolares como criterio de rendimiento académico. Recuperado en: http://www3.usal. es./inico/investigacion/jornadas/jornada2/ comunc/cl7.html

Castillo-Parra, G. Gómez, E. \& Ostrosky-Solis, F. (2009). Relación entre las funciones cognitivas y el nivel de rendimiento académico en niños. Neuropsicología, Neuropsiquiatría y Neurociencias, 9(1), 41-54.

Das, J. (1995). Some thought on two aspect of Vigotsky's work. Educacional Psychologist, 30, 93-97.

Gómez, L. Duarte, A. Merchán V. Aguirre D. \& Pineda D. (2007). Conciencia fonológica y comportamiento verbal en niños con dificultades de aprendizaje. Universitas Psychologica. Consultado en abril 272010. Recuperado en: http://www.scielo.org.co/
scielo.php?script=sci_arttext\&pid=S1657$92672007000300009 \&$ lang=pt.

Hernández-Sampieri. R., Fernández Callado, C. \& Batista, P. (2008). Metodología de la investigación. México: Mc Graw Hill.

Ivanovic, R., Ivanovic, D. (1988). Rendimiento Escolar y Estado Nutricional. En Ivanovic, D. Ivanovic, R. Middleton S. (Ed.). Rendimiento y deserción escolar: un enfoque multicausal (pp. 3-6). Santiago: Universidad de Chile, INTA.

Macizo, P., Bajo, T. \& Soriano, M. (2006) Memoria operativa y control ejecutivo: procesos inhibitorios en tareas de actualización y generación aleatoria. Psicothema, 18(001), 112-116.

Navarro, R. (2003). El rendimiento académico: Concepto, investigación y desarrollo. REICE - Revista Electrónica Iberoamericana sobre Calidad, Eficacia y Cambio en Educación, 1(2), 1-15.

Niebla, J. \& Hernández, L. (2007). Variables que inciden en el rendimiento académico de adolescentes mexicanos. Revista Latinoamericana de Psicología, 39(003), 487-501.

Paba, C., Lara, R. \& Palmezano, A. (2008) Estilos de aprendizaje y rendimiento académico en estudiantes universitarios. Revista de la Facultad de Ciencias de la Salud Universidad del Magdalena, 5(2).

Robledo, P. García, J. Diez, C. Álvarez, M. Murban, J. Caso, A. Fidalgo, R. Arias, O. \& Pacheco, D. (2010). Estilos de pensamiento y aprendizaje en estudiantes de magisterio y psicopedagogía: diferencias según curso y especialidad. Escritos de Psicología. Consultado en enero 13 de 2012. Recuperado de: http:// www.redalyc.org/src/inicio/ArtPdfRed. jsp?iCve $=271019808005$

Santrok, J. (2001). Psicología de la educación. México: MgGraw Hill. 
Schunk, D. H. (1997). Teorías del Aprendizaje. México: Pearson Educacion.

Siegel, L. \& Ryan, E. (1989). The development of working memory in normally achieving and subtypes of learning disabled children. Child Development, 60, 976-980.

Swanson, H. (2000). Are working memory deficits in readers whit learning disabilities hard to change? Journal of Learning Disabilities, 33, 551-567.
Wechsler, D. (2007). WISC - IV Escala de Wechsler de inteligencia para niños. Manual técnico. México: Manual Moderno.

Woolfolk, A. (1999). Psicología Educativa. México: Prentice Hall.

Zapata, L. De los Reyes, C. Lewis, S. \& Barceló, E. (2009). Memoria de trabajo y rendimiento académico en estudiantes de primer semestre de una Universidad de Barranquilla. Psicología desde el Caribe. Consultado en enero 13 de 2012. Recuperado de: http:// redalyc.uaemex.mx/src/inicio/ArtPdfRed. jsp?iCve=21311917005 